\title{
Improvement of MAEQ Method for Speech Enhancement in Noisy Environments
}

\author{
Hiroyuki Takamatsu a, ${ }^{\mathrm{a}}$, Yuto Tanakaa ${ }^{\mathrm{a}}$, Yoshihisa Nakatoh ${ }^{\mathrm{a}}$ \\ ${ }^{\text {a}}$ Kyushu Institute of Technology \\ 1-1, Sensui-cho, Tobata-ku, Kitakyusyu-shi, Fukuoka, 804-0015, Japan \\ *Corresponding Author: p349424h@mail.kyutech.jp
}

\begin{abstract}
It is hard to hear announced speech in noisy environments such as a running car. One of the techniques to improve the intelligibility of amplification sound in noisy environments is the MAEQ (Masking Adaptation Equalizer) method. The MAEQ method is a technique to improve intelligibility based on masking the amplification sound around the noise. However, speech made from the conventional MAEQ method is not clear in a low frequency.

In this paper, we improved the conventional MAEQ method and compared it with the conventional method and our improved method by speech intelligibility. In a band of $500-3 \mathrm{kHz}$, our proposed method is the conventional MAEQ method. In other bands, we limited the quantity of masking to $+7.31 \mathrm{~dB}$.

As a result from comparing the conventional MAEQ method in a noisy environment ( $60 \mathrm{dBA})$, in young adults, our proposed method improved by $11 \%$ for speech intelligibility. In elderly people, our proposed method improved by $17 \%$ for speech intelligibility. Our proposed method is more effective for elderly people than for young adults.
\end{abstract}

Keywords: amplification sound, announce speech, MAEQ method, noisy environment.

\section{Introduction}

In an extremely noisy environment, it is hard to hear amplification sound. For example, this happens in running cars and concourses. It is a phenomenon that announced speech happens because it is masked by the noise. One of the techniques to improve the intelligibility of the amplification sound in a noisy environment is the MAEQ (Masking Adaptation Equalizer) method.

In this paper, we improved the conventional MAEQ method and compared it with the conventional method and our improved method by speech intelligibility. In addition, we performed an evaluation experiment in elderly people with young adults and examined the tendency according to age.

\section{Improvement of the intelligibility by the MAEQ method}

Here, we describe the summary of the conventional MAEQ method and our proposed method.

\subsection{Summary of the conventional MAEQ method}

The MAEQ method is a technique to improve intelligibility based on masking the amplification sound around the noise. Specifically, the MAEQ method controls the volume and sound quality in consideration of auditory masking of amplification sound (maskee) by noise (masker). As for the precondition, the maskee is known, and the masker is unknown. At first, the flow of the processing calculates the level of the masker. Next, the quantity of masking $\mathrm{M}[\mathrm{dB}]$ is estimated for every critical band by equation (1). This is a function of level differences between the maskee $\mathrm{S}[\mathrm{dB}]$ and masker $\mathrm{N}[\mathrm{dB}]^{(1)}$. However, there are two conditions.

$$
\begin{aligned}
& \mathrm{M}=0.0072(\mathrm{~S}-\mathrm{N}) 2-0.53(\mathrm{~S}-\mathrm{N})+7.31[\mathrm{~dB}] \\
& \text { 1) } 50 \leqq \mathrm{~S} \leqq 90[\mathrm{~dB}], 30 \leqq \mathrm{~N} \leqq 90[\mathrm{~dB}] \\
& \text { 2) More than }(\mathrm{S}-\mathrm{N})=20[\mathrm{~dB}], \mathrm{M}=0[\mathrm{~dB}]
\end{aligned}
$$




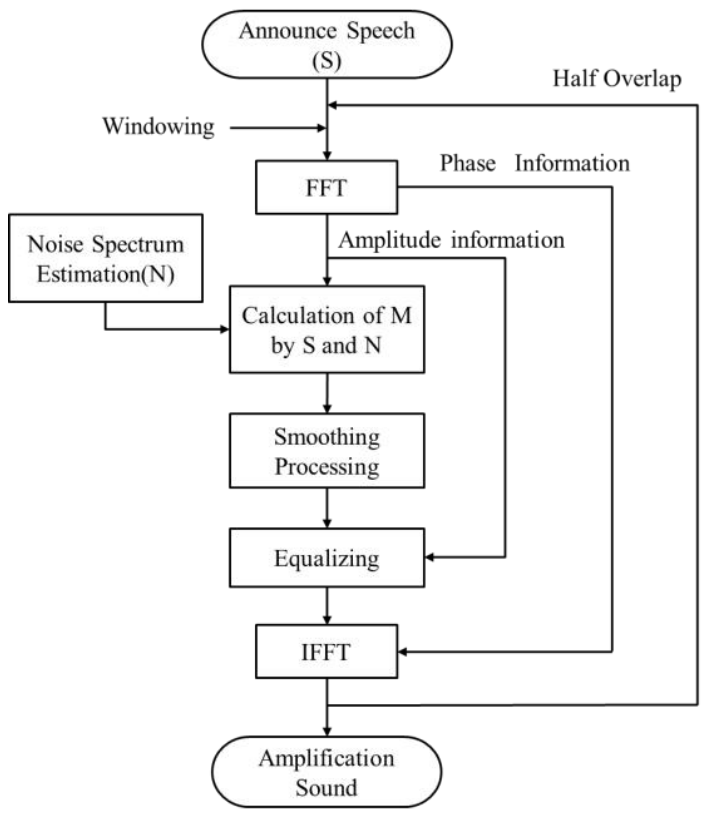

Fig. 1. Flow chart of the processing.

Expression (1) was similar to a multiple regression analysis about quantity of masking where a critical band noise was used to assume $1 \mathrm{kHz}$ central frequency given to a pure tone of $1 \mathrm{kHz}{ }^{(1)}$.

The precedent study says that we can apply expression (1) to all critical bands if we meet conditions 1) and 2) ${ }^{(1)}$. Condition 1) shows that the level of the maskee and masker limits the practical range in consideration of the reproduction level of the sound. Condition 2) shows that we do not perform equalization when there are level differences between the maskee and masker of more than $20 \mathrm{~dB}$.

Finally, the quantity of masking $\mathrm{M}$ equalizes it for all bands of the maskee after it was made smooth. The reason to perform smoothing is because it fluctuates the pettiness calculated quantity of masking. When we perform equalization without smoothing, the amplification sound is warped.

In this paper, we divided the sound of the $8 \mathrm{kHz}$ band (sampling frequency $16 \mathrm{kHz}$ ) into the critical band of 22 bands. We also performed smoothing with a moving average of $250 \mathrm{~ms}$ width. In addition, we assumed the upper limit of M $20 \mathrm{~dB}$ to prevent surplus equalization. In this way, the MAEQ method can clear up a sound depending on a characteristic of the noise.

Quantity of masking calculated by expression (1) is shown in Figure 2. The sound that we used is a word with four mora, "AKAGANE". The quantity of masking of Figure 2 fluctuates finely.

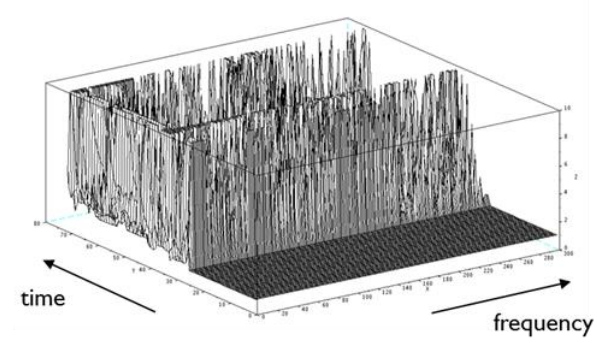

Fig. 2. Calculated quantity of masking.

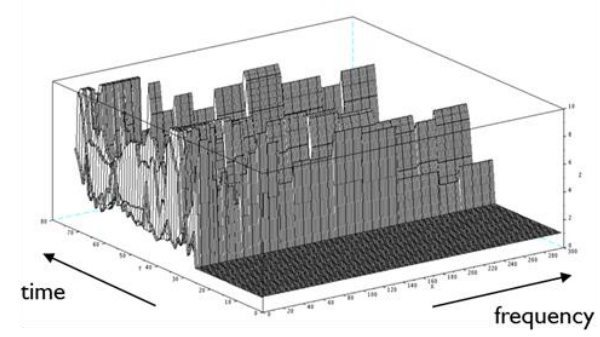

Fig. 3. Quantity of masking for every critical band.

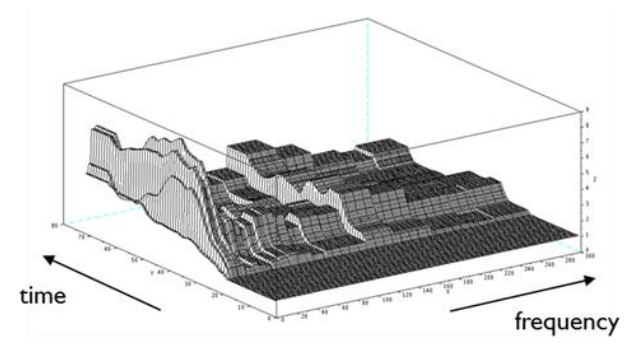

Fig. 4. Quantity of masking that performed smoothing in the time axis direction.

Figure 3 is the figure which calculated quantity of masking of Figure 2 for every critical band.

Figure 4 is the figure which performed smoothing of quantity of masking of Figure 3 in the time axis direction. This quantity of masking becomes more fluent than Figure 2. Quantity of masking of Figure 4 is the quantity of equalization.

\subsection{Improvement of the MAEQ method}

In this paper, we suggested the improved MAEQ method so that we may perform conventional MAEQ processing in the band of $500-3 \mathrm{kHz}$, and we confine equalization quantity to $+7.31 \mathrm{~dB}$ in the band. In other words our proposed method limits processing by one band. This is because the conventional MAEQ method may have the excessive equalization by the estimated error in the noise environment with big power in a considerably-low band. As a result, speech made from the conventional MAEQ method is not clear in a low frequency. 


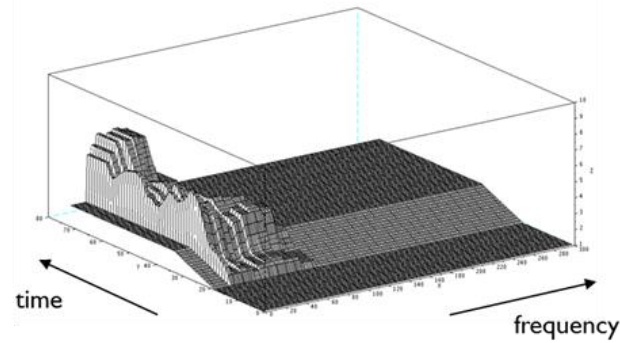

Fig. 5. Quantity of masking using the improved MAEQ method.

In addition, speech intelligibility is not improved, even if equalization does the conventional MAEQ method in a high band of more than $3 \mathrm{kHz}$. We confirmed this by a preliminary experiment.

Therefore, only the central band $(500-3 \mathrm{kHz})$ of the sound performed normal equalization, and we limited the equalization in the band which adversely affected intelligibility by surplus equalization. Therefore, improvement of speech intelligibility is expected.

Figure 5 is the quantity of masking using the improved MAEQ method. The sound that we used is the same as Figures 2-4. This quantity of masking is limited in comparison with Figure 4 in a band which is not 500-3 $\mathrm{kHz}$.

We inspected the usefulness of the improved MAEQ method by a speech intelligibility experiment. We equated the power of the sound which was equalized by the conventional MAEQ method with the power of the sound that we made equalization by the improved MAEQ method. Furthermore, we prepared to compare the sound (source signal) that did not perform equalization processing.

\section{Inspection of the improvement effect by speech intelligibility}

We inspected the usefulness of the improved MAEQ method by a speech-intelligibility experiment. Here, we describe the details of the speech-intelligibility experiment. Then, we describe the result and consideration.

\subsection{Experiment method}

We placed the speaker for sound presentation in the front of the subject who sat in the center of a soundproofing room, as is shown in Figure 6. Table 1 is a presentation of the sound pressure level in the head central location of the subjects. The level of source signal is $50 \mathrm{dBA}$ for the young adults and $60 \mathrm{dBA}$ for the elderly people.

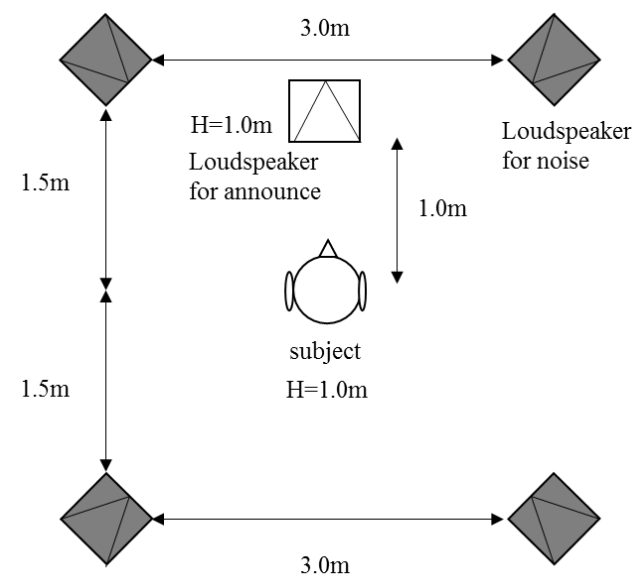

Fig. 6. Arrangement of loudspeakers.

The level of the noise is $55 \mathrm{dBA}$ (condition A), $60 \mathrm{dBA}$ (condition B), and $65 \mathrm{dBA}$ (condition $\mathrm{C}$ ). We located a speaker for noise presentation in four places surrounding the subject outwardly to simulate the environment of a running car. The noise used is the noise of a running car.

The subjects were five men with a normal hearing ability in their twenties and five elderly people. When the subjects head a noise, and they filled in the answer paper with the word that they heard.

We used the male sounds (mya) of a sound data set (FW7) from the year 2007 for word intelligibility examinations by NTT - Tohoku University. We extracted 180 Japanese words with four mora from a word list of Familiarity 1. We assigned 20 words for each of the three kinds of presentation sounds, the conventional MAEQ method, the improved MAEQ method, and the source signal. We replaced a word and tested the three kinds of conditions twice so that deflection would not be given to intelligibility by a word.

\subsection{Results and discussion}

Figure 7 is a comparison result of speech intelligibility in the young adults. As the noise level rose in the source signal, speech intelligibility decreased conspicuously. Particularly, speech intelligibility in Condition $\mathrm{C}$ was less than 20\%. In contrast, intelligibility of the MAEQ processing sound became more than $60 \%$ in all conditions. This is regarded as a difference in SNR of the noise for the sound. According to Figure 7, the improved MAEQ method improved intelligibility for the conventional MAEQ method $8 \%$ in Condition A, and $11 \%$ in Condition B. Thus, we were able to confirm the sound emphasis effect of the improved MAEQ method in the young adults. However, there were few differences of intelligibility between the improved 
MAEQ method and the conventional MAEQ method in Condition $\mathrm{C}$. Therefore, we think that the usefulness of the improved MAEQ method decreases when the SNR of a source signal and the noise are worse conditions than -15 dB.

Figure 8 is a comparison result of the speech intelligibility in the elderly people. As the noise level rose, like adults, in the case of the source signal, speech intelligibility decreased. However, the drop of intelligibility was calmer than the young adults. It is thought that the cause is because the level of source signal had a larger 10 $\mathrm{dB}$ than the young adults. Then, intelligibility of the MAEQ processing sound became more than $60 \%$ in all conditions. According to Figure 8, intelligibility of the improved MAEQ method became higher than the conventional MAEQ method in all conditions in the elderly people. Thus, we were able to confirm the sound emphasis effect of the improved MAEQ method in the elderly people. Specifically, intelligibility improved by $17 \%$ in Conditions $\mathrm{A}$ and $\mathrm{B}$, and improved by $5 \%$ in Condition $\mathrm{C}$.

Therefore, the improved MAEQ method is more effective for elderly people than young adults. Two factors were thought to have been the cause. For the first, a sound was cleared by the process of the improved MAEQ method. For the second, elderly people may be inferior to young adults in their ability to hear sounds of $500-3 \mathrm{kHz}$.

Table 1. Output sound pressure level from loudspeakers.

\begin{tabular}{|c|c|c|c|}
\hline Conditions & A & B & C \\
\hline Level of noise & $55 \mathrm{dBA}$ & $60 \mathrm{dBA}$ & $65 \mathrm{dBA}$ \\
\hline Level of source signal & \multicolumn{2}{|c|}{$\begin{array}{c}50 \mathrm{dBA} \text { (for young adults), } \\
60 \mathrm{dBA} \text { (for elderly people) }\end{array}$} \\
\hline
\end{tabular}

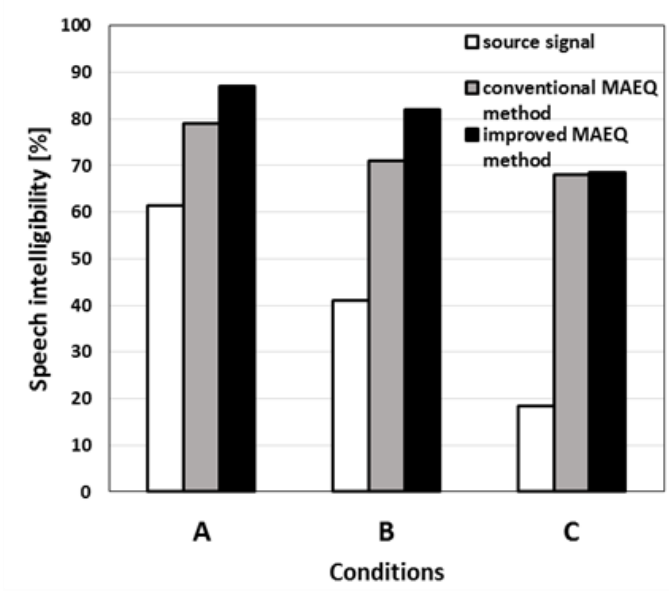

Fig. 7. Comparison with speech intelligibility in young adults.

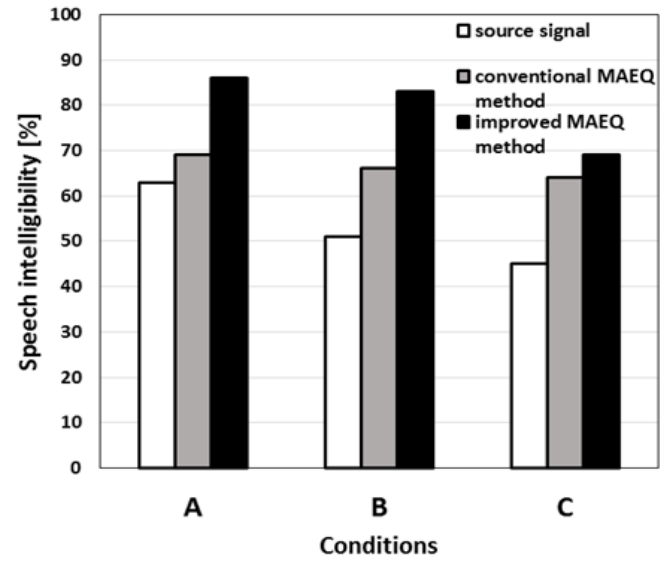

Fig. 8. Comparison with speech intelligibility in elderly people.

\section{Conclusions}

We improved the MAEQ method, which improved the articulacy of the amplification of sound in a noisy environment, and examined the improved effect of word intelligibility. As a result, the improved MAEQ method improved intelligibility for the conventional MAEQ method by $8 \%$ in Condition $\mathrm{A}$, and $11 \%$ in Condition $\mathrm{B}$ in the young adults. In addition, intelligibility was improved by $17 \%$ in Conditions $\mathrm{A}$ and $\mathrm{B}$, and improved by $5 \%$ in Condition $\mathrm{C}$ in the elderly people. Therefore, our proposed method is particularly effective for elderly people. We are going to experiment on an evaluation using various types of noise in the future. Furthermore, we are going to examine a new calculation method of the masking quantity to improve intelligibility of the amplification of sound.

\section{References}

(1) A. Murase, K. Nakamura and K. Iida, "Adaptive Tone Control Method According to Masking Level Caused by Noise," Autumn Meeting of The Acoustical Society of Japan, pp. 523-524, 1997.

(2) A. Murase, K. Nakamura and K. Iida, "Improvement of sentence intelligibility by the noise adaptation sound reproducing method," Autumn Meeting of The Acoustical Society of Japan, pp. 459-460, 2000.

(3) Y. Nomura, J. LU, H. Sekiya and T. Yahagi, "Speech/noise-dominant decision regardless of SNR for speech enhancement," Information and Communication Engineers Technical Report of IEICE, pp. 29-34, 1997. 